\title{
STRUCTURE FORMATION:
}

\section{MODELS, DYNAMICS AND STATUS}

\author{
T. PADMANABHAN \\ Inter-University Centre for Astronomy and Astrophysics, \\ Post Bag 4, Ganeshkhind, Pune - 411 007, INDIA. \\ Email: paddy@iucaa.ernet.in
}

\section{Recipe for the Universe}

All the popular models for structure formation are based on three key ingredients: (a) a model for the background universe (b) some mechanism for generating small perturbations in the early universe and (c) specification of the nature of the dark matter.

The background universe is usually taken to be a Friedmann model with an expansion factor $a(t)$. Such a model is completely specified if the composition of the energy density and the Hubble constant are specified. We will take $H_{0}=100 \mathrm{~h} \mathrm{kms}^{-1} \mathrm{Mpc}^{-1}$ and express the energy density of the various species in terms of the critical density $\rho_{c}=\left(3 H_{0}^{2} / 8 \pi G\right)=$ $1.88 h^{2} \times 10^{-29} \mathrm{~g} \mathrm{~cm}^{-3}$, by writing $\rho_{i}=\Omega_{i} \rho_{c}$ for the $i^{t h}$ species. From various observations, we can impose the following constraints: (i) $0.011 h^{-2} \lesssim \Omega_{B} \lesssim$ $0.016 h^{-2}$ (ii) $\Omega_{\text {vac }} \lesssim 0.8$ (iii) $\Omega_{\text {lum }} \simeq 0.007 h$ (iv) $\Omega_{R}=4.85 h^{-2} \times 10^{-5}$ (v) $\Omega_{\text {total }} \equiv \Omega \gtrsim 0.3$. Theoretical models strongly favor $\Omega=1$ and it is usual to invoke either a cosmological constant and/or nonbaryonic dark matter to achieve this (e.g. Carney \& Latham 1987, Pagel 1991). We shall denote by $\Omega_{D M}$ the total contribution due to all nonbaryonic energy densities.

Models for structure formation also need to assume that small perturbations in the energy density existed at very early epochs. These perturbations can then grow via gravitational instability leading to the structures we see today. In most of the models these perturbations are generated by processes which are supposed to have taken place in the very early universe (say, at $z \gtrsim 10^{18}$ ). Inflationary models - which are probably the most successful ones in this regard - can produce density perturbations with an initial power spectrum $P_{\text {in }}(k) \simeq A k$. Since each logarith- 
mic interval in $k$ space will contribute to the energy density an amount $\triangle_{\rho}^{2}(k) \equiv d \sigma^{2} / d(\ln k)=\left(k^{3} P(k) / 2 \pi^{2}\right)$ we find that $\triangle_{\rho}^{2} \propto k^{4}$ for $P \propto k$. The contribution to the gravitational potential from the same range will be $\triangle_{\varphi}^{2}=\triangle_{\rho}^{2}\left(9 H_{0}^{4} / 4 k^{4} a^{2}\right)$ which is independent of $k$ if $\triangle_{\rho}^{2} \propto k^{4}$. Such a "scale-invariant" spectrum is produced in some other seeded models as well. All these models need to be fine-tuned to keep the amplitude $A$ of the fluctuations small up to, say, $z \gtrsim 10^{3}$.

Given a Friedmann model with small inhomogeneities described by a power spectrum $P\left(k, z_{\text {in }}\right)$ at a high redshift $z=z_{\text {in }}$, we can predict unambiguously the power spectrum $P\left(k, z_{D}\right)$ at the epoch of decoupling $z_{D} \approx 10^{3}$. This is because the perturbations at all relevant scales are small at $z \gtrsim z_{D}$ and we can use linear perturbation theory during this epoch. The shape of the spectrum at $z=z_{D}$ will not be a pure power law since the gravitational amplifiction is wavelength-dependent. In general, the power at small scales is suppressed (relative to that at large scales) due to various physical processes and the exact shape of the spectrum at $z=z_{\mathrm{D}}$ depends on the kind of dark matter present in the universe. In a universe dominated by "hot dark matter" particles of mass $m \simeq 30 \mathrm{eV}$, the power per logarithmic interval in $k$-space, $\triangle(k)=\left(k^{3} P(k) / 2 \pi^{2}\right)^{1 / 2}$, is peaked at $k=k_{\max } \equiv 0.11$ $\mathrm{Mpc}^{-1}(\mathrm{~m} / 30 \mathrm{eV})$ and falls exponentially for $k>k_{\max }$. Hence, in these models, the scale $k=k_{\max }$ will go nonlinear first and smaller structures have to form by fragmentation. If the universe is dominated by "cold dark matter" particles with mass $m \gtrsim 35 \mathrm{GeV}$, then $\Delta(k)$ is a gently increasing function of $k$ for small $k$. If we set $P(k) \propto k^{n}$ locally, the index $n$ changes from 1 at $k^{-1} \gtrsim 200 h^{-1} \mathrm{Mpc}$ to $0 \mathrm{at} k^{-1} \simeq 10 h^{-1} \mathrm{Mpc}$ and to about $(-2)$ at $k^{-1} \simeq 1 h^{-1}$ Mpc. In such models small scales will go nonlinear first and the structure will develop hierarchically (e.g. Padmanabhan 1993).

The situation is more complicated if two different kinds of dark matter are present or if the cosmological constant is nonzero. The presence of the cosmological constant adds to the power at large scales but suppresses the growth of perturbations at small scales. Similar effects take place if a small fraction of the dark matter is hot and the bulk of it is cold (eg. $\Omega_{\mathrm{HDM}} \simeq 0.2, \Omega_{C D M} \simeq 0.8$ ). In both the cases there will be more power at large scales and less power at small scales, compared to standard CDM model. The spectrum $\triangle(k)$ is still a gently increasing function of $k$ and small scales go nonlinear first.

The fact, that one can compute the power spectrum at $z \simeq z_{D}$ analytically, allows one to predict large scale anisotropies in CMBR unambiguously in any given model. Comparing this prediction with the anisotropy observed by COBE one can fix the amplitude $A$ of the power spectrum. For a wide class (Padmanabhan 1992) of the models, $\triangle(k) \cong 10^{-3}(k L)^{2}$ with $L \simeq(24 \pm 4) h^{-1} \mathrm{Mpc}$ for $k^{-1} \gtrsim 80 h^{-1} \mathrm{Mpc}$. For CDM like mod- 
els the function $\triangle(k)$ flattens out at larger $k$ and is about unity around $k^{-1} \simeq 8 h^{-1}$ Mpc. In pure HDM models, $\triangle(k)$ has a maximum value of $\triangle_{m} \simeq 0.42 h^{-2}(m / 30 \mathrm{eV})^{2}$ at $k_{m} \simeq 0.11 \mathrm{Mpc}^{-1}(\mathrm{~m} / 30 \mathrm{eV})$ and decreases exponentially at $k \gtrsim k_{m}$.

The evolution of the power spectrum after decoupling (for $z<z_{\mathrm{D}}$ ) is more difficult to work out theoretically. In general, the power spectrum grows in amplitude (preserving the shape), as long as the perturbations are small (e.g. Padmanabhan 1993). In this case, we can write $\triangle(k, z)=$ $\left[f(z) / f\left(z_{D}\right)\right] \triangle\left(k, z_{D}\right)$ for $z<z_{D}$. For example, in CDM models with $\Omega=$ $1, f(z)=(1+z)^{-1}$; thus $\triangle(k)$ grows by a factor $10^{3}$ at all scales between the epoch of decoupling $\left(z_{D} \simeq 10^{3}\right)$ and the present epoch $(z=0)$, if we assume that linear theory is valid at all scales. The resulting $\triangle_{0}(k)$, obtained by linear extrapolation, is often used to specify the properties of the models. This spectrum correctly describes the power at large scales (say, $k^{-1} \gtrsim 30 h^{-1} \mathrm{Mpc}$ ) where $\triangle_{0} \lesssim 0.1$. The "density contrast" $\sigma(R)$ measures the rms fluctuations in mass within a randomly placed sphere of radius $R$; up to factors of order unity, $\sigma(R) \simeq \triangle\left(k \simeq R^{-1}\right)$ in hierarchical models. For most of the, COBE normalized, CDM-like models $\sigma(R) \approx 1$ around $R \approx 8 h^{-1} M p c$. Clearly linear theory cannot be trusted at smaller scales.

There are two major difficulties in understanding the physics at these small scales. Firstly, the true power $\triangle_{\text {true }}(k)$ of dark matter will be larger than $\triangle_{0}(k)$ due to nonlinear effects which are difficult to model analytically. Since dark matter particles interacts only through gravity, it is, of course, possible to study the formation of dark matter structures by numerical simulations. But to gain insight into the dynamics, it will be helpful to have simple analytic models explaining the $\mathrm{N}$-body results.

Secondly, it is important to understand gas dynamical processes before one can compare theory and observations at small scales. Since baryons can dissipate energy and sink to the minima of the dark matter potential wells, the statistical properties of visible galaxies and dark matter halos could be quite different. The situation is further complicated by the fact that in hierarchical models, considerable amounts of merging takes place at small scales. It is usual to quantify our ignorance at these scales by a 'bias' (acronym for 'Basic Ignorance of Astrophysical Scenarios') factor $b$ and write $\xi_{\text {gal }}(r)=b^{2} \xi_{\text {mass }}(r)$. Such a parametrization is useful only if $b$ is independent of scale and morphology of galaxies. This seems to be somewhat unlikely. Since small scale observations are based on galactic properties, while theoretical calculations usually deal with underlying mass distribution, any scale (or morphology) dependence of $b$ could play havoc with predictive power of the theory.

Recently some amount of progress has been achieved as regards the first 
aspect, viz, understanding nonlinear clustering of dark matter (Hamilton et al. 1991, Nityananda \& Padmanabhan 1994, Bagla \& Padmanabhan 1993, Padmanabhan et al. 1995). This approach is based on the relationship between the mean correlation function $\bar{\xi}(x, a)$ and the mean relative pair velocity $v(x, a)$. These quantities are related by an exact equation.

$$
\frac{\partial F}{\partial A}-h(A, X) \frac{\partial F}{\partial X}=0
$$

where $F=\ln \left[x^{3}(1+\bar{\xi})\right], A=\ln a, X=\ln x$ and $h=-(v / \dot{a} x)$. The characteristics of this equation shows that, as the evolution proceeds, power from a large scale $l$ is transferred to smaller scales up to $x=l(1+\bar{\xi})^{-1 / 3}$. By analyzing the behavior of $h$, it is possible to express $\bar{\xi}(x, a)$ in terms of the mean correlation function in the linear theory, $\bar{\xi}_{L}(l, a)$. It turns out that: $\bar{\xi}(a, x)=Q\left[\bar{\xi}_{L}(a, l)\right]^{n}$ with $l^{3}=x^{3}(1+\bar{\xi})$ where $Q=n=1$ for $\bar{\xi}_{L} \leq 1.2 ; Q=0.7, n=3$ for $1.2 \leq \bar{\xi}_{L} \leq 6.5$ and $Q=11.7, n=1.5$ for $\bar{\xi}_{L} \geq 6.5$. This relation shows that $\bar{\xi}$ is steeper than $\bar{\xi}_{L}$.

Unfortunately, no such simple pattern exists in the dynamics of baryons coupled to dark matter. The gas dynamical processes introduce several characteristic scales into the problem and the evolution becomes quite complicated. The only reliable way of probing these systems seems to be through massive hydro simulations which are still at infancy.

It is clear from the above discussions that our theoretical understanding is best at large scales $\left(k^{-1} \gtrsim 30 h^{-1} \mathrm{Mpc}\right)$ where linear theory is valid, $\triangle_{0}(k)$ is well determined and baryonic astrophysical processes are not important. At the intermediate scales $\left(3 h^{-1} \mathrm{Mpc} \lesssim k^{-1} \lesssim 30 h^{-1} \mathrm{Mpc}\right)$, it is not too difficult to understand the dark matter dynamics by some approximation but the baryonic physics begins to be nontrivial. At still smaller scales, $\left(k^{-1} \lesssim 3 h^{-1} \mathrm{Mpc}\right)$ there is considerable uncertainty in our theoretical predictions. We shall now turn to the observational probes of the power spectrum at different scales.

\section{Probing the power spectrum}

One of the direct ways of constraining the models is to estimate the density contrast $\sigma_{\text {obs }}(R)$ from observations at different scales and compare it with the theoretically predicted values. Fortunately, we now have observational probes covering four decades of scales from $10^{-1} \mathrm{Mpc}$ to $10^{3} \mathrm{Mpc}$. We shall discuss the probes of different scales in decreasing order.

\subsection{NEAR HORIZON SCALES: $(300-3000) \mathrm{H}^{-1} \mathrm{MPC}$}

These scales are so large that the best way to probe them is by studying the MBR anisotropy at angular scales which correspond to these linear 
scales. Since a scale $L$ subtends an angle $\theta(L) \cong 1^{\circ}\left(L / 100 h^{-1} \mathrm{Mpc}\right)$ at $z \simeq z_{D}$, the $(\triangle T / T)$ observations at $\left(3^{\circ}-30^{\circ}\right)$ probe these scales. The COBE-DMR observations (Smoot et al. 1992) of $(\triangle T / T)_{\text {rms }}$ and $(\triangle T / T)_{Q}$ allow one to obtain the following conclusions: (i) $\sigma\left(10^{3} h^{-1} \mathrm{Mpc}\right) \simeq 5 \times 10^{-4}$ (ii) The power spectrum at large scales is consistent with $P_{\text {in }}(k) \simeq A k$ and, if we take $\Omega=1$, then $A^{1 / 4} \cong(24 \pm 4) h^{-1} \mathrm{Mpc}$ (iii) In this range, $\sigma(R) \cong\left(24 \pm 4 h^{-1} \mathrm{Mpc} / R\right)^{2}$.

\subsection{VERY LARGE SCALES : $(80-300) H^{-1}$ MPC}

\subsubsection{CMBR probes:}

These scales $\operatorname{span}\left(0.8^{\circ}-3^{\circ}\right)$ in the sky at $z \simeq z_{D}$. Several ground based and balloon-borne experiments to detect anisotropy in MBR probe this scale. For example, the UCSB South Pole experiment (Schuster et al. 1993) has reported a preliminary 'detection' of $(\triangle T / T) \simeq 10^{-5}$ at $1.5^{\circ}$ scale, and a $95 \%$ confidence level bound of $(\triangle T / T)<5 \times 10^{-5}$. This translates into the constraint of $\sigma\left(10^{2} h^{-1} \mathrm{Mpc}\right) \lesssim 5 \times 10^{-2}$.

The angular anisotropy of CMBR is dominated by the gravitational potential wells of dark matter at large scales. However, at $\theta \simeq 1^{\circ}$ baryonic process affect the pattern of anisotropy significantly. The precise determination of degree scale anisotropy can, therefore, help in distinguishing between different models (White, Scott \& Silk 1994).

\subsubsection{Galaxy surveys:}

Some galaxy surveys, notably CfA2 survey and pencil-beam surveys probe scales which are about $10^{2} h^{-1} \mathrm{Mpc}$ in depth (Broadhurst et al. 1990, Vogeley et al. 1992). Unfortunately, the statistics at these large scales is not good enough for one to obtain $\sigma(R)$ directly from these surveys.

\subsection{LARGE SCALES : $(40-80) H^{-1}$ MPC}

\subsubsection{CMBR probes:}

The scales correspond to $\theta_{M B R} \simeq\left(24^{\prime}-48^{\prime}\right)$ and are probed by the experiments looking for small angle anisotropies in MBR. The claimed detection (Cheng et al. 1994) by MIT-MASM of $(\triangle T / T) \cong(0.5-1.9) \times 10^{-5}$ at $\theta \simeq 28^{\prime}$, if confirmed, will give a bound of $\sigma\left(50 h^{-1} \mathrm{Mpc}\right) \lesssim 0.3$.

\subsubsection{Galaxy Surveys:}

Several galaxy surveys, in particular the IRAS-QDOT and APM surveys, give valuable information about this range (Rowan-Robinson et al. 1990, Efstathiou et al. 1990, Saunders et al. 1991). The angular correlation of galaxies, measured by APM survey is $\omega(\theta) \simeq(1-5) \times 10^{-3}$ at $\theta \simeq 14^{\circ}$. This corresponds to $\sigma\left(50 h^{-1} \mathrm{Mpc}\right) \cong 0.2$. What is more important, these surveys 
can provide valuable information about the shape of the power spectrum in this range if we assume that galaxies faithfully trace the underlying mass distribution.

\subsubsection{Large scale velocity field:}

Using distance indicators which are independent of Hubble constant, it is possible to determine the peculiar velocity field $v(R)$ of galaxies up to about $80 h^{-1} \mathrm{Mpc}$ or so. The motion of these galaxies can be used to map the underlying gravitational potential at these scales. Careful analysis of observational data shows (e.g. Dekel 1994) that $v\left(40 h^{-1} \mathrm{Mpc}\right) \simeq(388 \pm 67)$ $\mathrm{kms}^{-1}$ and $v\left(60 h^{-1} \mathrm{Mpc}\right) \simeq(327 \pm 82) \mathrm{kms}^{-1}$. From these values it is possible to deduce that $\sigma\left(50 h^{-1} \mathrm{Mpc}\right) \simeq 0.2$. These observations also allow us to determine the value of the parameter $\left(\Omega^{0.6} / b_{\text {IRAS }}\right)$ where $b_{\text {IRAS }}$ is the bias factor with respect to IRAS galaxies. One finds that $\left(\Omega^{0.6} / b_{\text {IRAS }}\right)=$ $1.28_{-0.59}^{+0.75}$ which implies that if $\Omega=1$, then $b_{\text {IRAS }}=0.78_{-0.29}^{+0.66}$ and if $b_{\text {IRAS }}=$ 1 then $\Omega=1.51_{-0.97}^{+1.74}$.

\subsubsection{Clusters and voids:}

The cluster-cluster correlation function and the spectrum of voids in the universe can, in principle, tell us something about these scales. Unfortunately, the observational uncertainties are so large that one cannot yet make quantitative predictions.

\subsection{INTERMEDIATE SCALES : $(8-40) H^{-1}$ MPC}

\subsubsection{Galaxy Surveys:}

The galaxy-galaxy correlation function $\xi_{\mathrm{gg}} \cong\left[r / 5.4 h^{-1} \mathrm{Mpc}\right]^{-1.8}$ is fairly well determined at these scales. Direct observations suggest that $\sigma_{\text {gal }}\left(8 h^{-1}\right.$ $\mathrm{Mpc}) \simeq 1$ but the $\sigma_{\mathrm{DM}}$ and $\sigma_{\mathrm{gal}}$ at these scales can be quite different because of possible biasing.

\subsubsection{Cluster Surveys:}

There have been several attempts to determine the correlation function of clusters of different classes. It is generally believed that $\xi_{\mathrm{cc}} \simeq(r / L)^{-1.8}$ with $L \simeq 25 h^{-1} \mathrm{Mpc}$. The index $n=1.8$ is fairly well determined though the scale $L$ is not; in fact, $L$ seems to depend on the richness class of the cluster. The quantity $\left(\xi_{\mathrm{cc}} / \xi_{\mathrm{gg}}\right)^{1 / 2}$ can be thought of as measure of the relative bias between cluster and galaxy scales. Observations suggest (Dalton et al. 1992, Nicol et al. 1992, Bahcall \& West 1992, Postman et al. 1992) that this quantity depends on the cluster class and varies in the range $(2-8)$. The observational uncertainties are still quite large for this quantity to be of real use; but if the observations improve we will have valuable information from $\xi_{c c}$. 


\subsubsection{Abundance of rich clusters:}

The scale $R=8 h^{-1} \mathrm{Mpc}$ contain a mass of $1.2 \times 10^{13} \Omega h_{50}^{-1} M_{\odot}$. When this scale becomes nonlinear, it will reach an overdensity of about $\delta \simeq$ 178 , or - equivalently - it will contract to a radius of $R_{f} \simeq\left(8 h^{-1} \mathrm{Mpc}\right)$ $/(178)^{1 / 3} \simeq 1.5 h^{-1} \mathrm{Mpc}$. A mass of $10^{15} M_{\odot}$ in a radius of $1.5 \mathrm{Mpc}$ is a good representation of Abell clusters we see in the universe. This implies that the observed abundance of Abell clusters can be directly related to $\sigma\left(8 h^{-1} \mathrm{Mpc}\right)$. Several people have attempted to do this (White et al. 1993); the final results vary depending on the modeling of Abell clusters, and give $\sigma\left(8 h^{-1}\right.$ $\mathrm{Mpc}) \simeq(0.5-0.7)$. Since $\sigma_{\mathrm{gal}}\left(8 h^{-1} \mathrm{Mpc}\right) \simeq 1$, this shows that $b \simeq(1.23-2)$ at $8 h^{-1} \mathrm{Mpc}$.

It is possible to give this argument in a more general context (Subramanian \& Padmanabhan 1994). Suppose that the contribution to critical density from collapsed structures with mass larger than $M$ is $\Omega(M)$, at a given redshift $z$. Then one can show that

$$
\Omega(M)=\operatorname{erfc}\left[\frac{\delta_{c}(1+z)}{\sqrt{2} \sigma_{0}(M)}\right]
$$

where $\delta_{c}=1.68$ and $\operatorname{erfc}(\mathrm{x})$ is the complementary error function. The Abell clusters (at $z=0)$ contribute in the range $\Omega \simeq(0.001-0.02)$. Even with such a wide uncertainty, we get $\sigma_{\text {clus }} \simeq(0.5-0.7)$.

\subsection{SMALL SCALES : $(0.05-8) H^{-1}$ MPC}

These scales correspond to structures with $M_{\text {smooth }} \simeq\left(3 \times 10^{8}-1.2 \times\right.$ $\left.{ }^{10} 0^{15}\right) \Omega h_{50}^{-1} M_{\odot}$ and we have considerable amount of observational data covering these scales. Unfortunately, it is not easy to make theoretical predictions at these scales because of nonlinear, gas dynamical, effects.

\subsubsection{Epoch of galaxy formation:}

Observations indicate that galaxy-like structures have existed even at $z \simeq 3$. This suggests that there must have been sufficient power at small scales to initiate galaxy formation at these high redshifts. Unfortunately, we do not have reliable estimate for the abundance of these objects at these redshifts and hence we cannot directly use it to constrain $\sigma(R)$.

\subsubsection{Abundance of quasars:}

The luminosity function of quasars is fairly well determined up to $z \approx 4$. If the astrophysical processes leading to quasar formation are known, then the luminosity function can be used to estimate the abundance of host objects at these redshifts. Though these processes are somewhat uncertain, most 
of the models for quasar formation suggest that we must have $\sigma\left(0.5 h^{-1}\right.$ $\mathrm{Mpc}) \gtrsim 3$.

\subsubsection{Absorption systems:}

The universe at $1 \lesssim z \lesssim 5$ is also probed by the absorption of quasar light by intervening objects. These observations suggest that there exist significant amounts of clumped material in the universe at these redshifts with neutral hydrogen column densities of $N_{\mathrm{HI}} \simeq\left(10^{15}-10^{22}\right) \mathrm{cm}^{-2}$. We can convert these numbers into abundances of dark matter halos by making some assumptions about this structure. We find that (Subramanian \& Padmanabhan 1994) in the redshift range of $z \simeq(1.7-3.5)$ damped Lyman alpha systems contribute a fractional density of $\Omega_{L y} \simeq(0.06-0.23)$. This would require $\sigma\left(10^{12} M_{\odot}\right) \simeq(3-4.5)$.

\subsubsection{Gunn-Peterson bound:}

While we do see absorption due to clumped neutral hydrogen, quasar spectra do not show any absorption due to smoothly distributed neutral hydrogen. Since the universe became neutral at $z \lesssim z_{D} \simeq 10^{3}$, and since galaxy formation could not have made all the neutral hydrogen into clumps, we expect the IGM to have been ionized sometime during $5 \lesssim z \lesssim 10^{3}$. It is not clear what is the source for these ionizing photons. Several possible scenarios (quasars, massive primordial stars, decaying particles etc.) have been suggested in the literature though none of these appears to be completely satisfactory. In all these scenarios, it is necessary to form structures at $z \gtrsim 5$ so that an ionizing flux of about $J=10^{-21} \mathrm{ergs} \mathrm{cm}^{-2} \mathrm{~s}^{-1} \mathrm{~Hz}^{-1} \mathrm{sr}^{-1}$ can be generated at these epochs. Once again, it is difficult to convert this constraint into a firm bound on $\sigma$ though it seems that $\sigma\left(0.5 h^{-1} \mathrm{Mpc}\right) \gtrsim 3$ will be necessary.

\section{Gravitational lensing and large scale structure}

In the above discussion we have not taken into consideration the constraints imposed by gravitational lensing effects on the structure formation models. This aspect will be discussed in detail in the other articles in this volume; here we shall contend ourselves with a brief mention of the possibilities.

Gravitational lensing probes the gravitational potential directly and can provide valuable information at very different scales. At the largest scales $\left(R \simeq 10^{3} \mathrm{Mpc}\right.$ ) lensing can be used to probe the geometry of the universe. For example, it is possible to put firm bounds on the energy contributed by cosmological constant from such considerations.

At intermediate scales ( $R \simeq 50 \mathrm{Mpc}$ ) lensing has the potential of providing information about the power spectrum of fluctuations which are in 
the quasilinear phase. In principle the distortion of images can be inverted to obtain this information, though in practice this is extremely difficult.

At smaller scales, the "weak lensing" - leading to arcs and arclets at cluster scales - is already providing a clue to the mapping of dark matter distribution in clusters. On the other hand, direct optical and X-ray observations provide us information about the distribution of visible matter in clusters. The combination of these techniques should give us valuable information as regards the dynamical processes which separated baryons from dark matter.

At still smaller scales, galactic potentials have the capacity to produce multiple images of distant sources. The statistics of these multiple images depends crucially on the core radii of the galaxies, which in turn depends sensitively on the structure formation models. The absence of significant number of multiple images with large angular separations puts severe constraints on models for structure formation. The analytic modeling of nonlinear dark matter clustering described earlier could be used to strengthen these constraints still further.

\section{Scorecard for the models}

The simplest models one can construct will contain a single component of dark matter, either cold or hot. Such models are ruled out by the observations. The HDM models, normalized to COBE result will have maximum power of $\triangle_{m} \cong 0.42 h^{-2}(m / 30 \mathrm{eV})^{2}$ at $k=k_{m}=0.11 \mathrm{Mpc}^{-1}(\mathrm{~m} / 30 \mathrm{eV})$. In such a case, structures could have started forming only around $\left(1+z_{c}\right) \cong$ $\left(\triangle_{m} / 1.68\right) \cong h_{50}^{-2}(m / 30 e V)^{2}$ or at $z_{c} \cong 0$. We cannot explain a host of high- $z$ phenomena with these models. The pure CDM models face a different difficulty. These models, normalized to COBE, predict $\sigma_{8} \simeq 1$, which is too high compared to the bounds from cluster abundance. When nonlinear effects are taken into account, one obtains $\xi_{\mathrm{gg}} \propto r^{-2.2}$ for $h=0.5$ which is too steep compared to the observed value of $\xi_{\mathrm{gg}} \propto r^{-1.8}$. In other words, CDM models have wrong shape for $\xi(r)$ to account for the observations.

The comparison of the CDM spectrum with observations suggests that we need more power at large scales and less power at small scales. This is precisely what happens in models with both hot and cold dark matter or in models with nonzero cosmological constant. These models have been extensively studied during the last few years, and they fare well as far as large and intermediate scale observations are concerned. However, they have considerably less power at small scales compared to CDM model. As a result, they do face difficulties (Subramanian \& Padmanabhan 1994) in explaining the existence of high redshift objects like quasars. For example, a model with $30 \% \mathrm{HDM}$ and $70 \% \mathrm{CDM}$ will have $\sigma_{0.5} \simeq 1.5$; to explain 
the abundance of quasars comfortably one needs $\sigma_{0.5} \simeq 3.0$. To explain the abundance of damped Lyman alpha systems one requires still larger values of about $\sigma_{0.5} \approx 4$ or so. Demanding that $\sigma\left(10^{12} M_{\odot}\right)>2$ [which is equivalent to saying that $10^{12} M_{\odot}$ objects must have collapsed at a redshift of $\left.z_{12}=(2 / 1.68)-1 \simeq 0.2\right]$ will completely rule out this model. Similar difficulties exist in models with cosmological constant. Notice that all models are normalized using COBE results at very large scales. Hence the severest constraints are provided by observations at smallest scales, since the "lever-arm" is longest in that case.

The comparison of models show that it is not easy to accommodate all the observations even by invoking two components to the energy density. (These models also suffer from serious problems of fine-tuning). By and large, the half-life of such quick-fix models seem to be about 2-3 years. One is forced to conclude that to make significant progress it is probably necessary to perform a careful, unprejudiced analysis of: (a) large scale observational results and possible sources of error and (b) small scale baryonic astrophysical processes.

\section{References}

Bagla, J.S. \& Padmanabhan, T., 1993, IUCAA preprint 22/93

Bahcall, N. \& West, M., 1992, ApJ, 270, 70

Broadhurst, T., et al., 1990, Nature, 343, 726

Carney, B.W. \& Latham, D.W., 1987, Dark Matter in the Universe, eds. J. Kormendy, \& G.R. Knapp (Dordrecht: Reidel) 39

Cheng, E.S., et al., 1994, ApJL, 422 L37

Dalton, G.D., et al., 1992, ApJL, 390, L1

Dekel, A., 1994, ARA\&A, 32, 371

Efstathiou, G., et al., 1990, MNRAS, 247, 10p

Hamilton, A.J.S., et al., 1991, ApJL, 374, L1

Nicol, R.C., et al., 1992, MNRAS, 255, 21p

Nityananda, R. \& Padmanabhan, T., 1994, MNRAS,271, 976

Padmanabhan, T., Narasimha, D., 1992, MNRAS, 259, 41P

Padmanabhan, T., 1993, Structure Formation in the Universe, (Cambridge: Cambridge University Press) chapter 4

Padmanabhan, T., et al., 1995, Princeton preprint astro-ph 9506051

Pagel, B.E.J., Phys Scr, 1991, T36, 7

Postman, M., et al., 1992, ApJ, 384, 404

Rowan-Robinson, M., et al., 1990, MNRAS, 247, 1

Saunders, W., et al., 1991, Nature, 349, 32

Schuster, J., et al., 1993, ApJL, 412, L47

Smoot, G.F., et al., 1992, ApJL, 396, L1

Subramanian, K., \& Padmanabhan, T., 1994, IUCAA preprint 5/94

Vogeley, M.S., et al., 1992, ApJL, 391, L5

White, S.D.M., et al., 1993, MNRAS, 262, 1023

White, M., Scott, D., \& Silk, J., 1994, ARA\&A, 32, 319 